Research Paper

\title{
Significant benefits of adding neoadjuvant chemotherapy before concurrent chemoradiotherapy for locoregionally advanced nasopharyngeal carcinoma: a meta-analysis of randomized controlled trials
}

\author{
Mengmeng Wang ${ }^{1}$, Huimin Tian ${ }^{1}$, Gang Li $^{2}$, Tingwen Ge$^{1}$, Yudi Liu ${ }^{1}$, Jiuwei Cui ${ }^{1}$, \\ Fujun $\operatorname{Han}^{1}$ \\ ${ }^{1}$ Cancer Center, The First Hospital of Jilin University, Changchun, China \\ ${ }^{2}$ Department of Thyroid and Breast Surgery, No.458 Hospital of People's Liberation Army, Guangzhou, China \\ Correspondence to: Fujun Han, email: fujun_han@aliyun.com \\ Keywords: adjuvant chemotherapy, concurrent chemoradiotherapy, meta-analysis, nasopharyngeal carcinoma, neoadjuvant \\ chemotherapy \\ Received: January 21, 2016 \\ Accepted: May 30, 2016 \\ Published: June 23, 2016
}

\section{ABSTRACT}

Purpose: We did a meta-analysis to compare the efficacy and safety of neoadjuvant chemotherapy (NACT) followed by concurrent chemoradiotherapy (CCRT) versus CCRT with or without adjuvant chemotherapy (AC) for patients with locoregionally advanced nasopharyngeal carcinoma based on randomized controlled trials.

Methods: We searched PubMed, Embase, Web of Science, ClinicalTrials.gov, Chinese National Knowledge Infrastructure, and meeting proceedings of major relevant conferences to identify published and unpublished randomized controlled trials. Progression-free survival (PFS) was the primary endpoint.

Results: This meta-analysis included 9 randomized clinical trials with 2215 patients. NACT followed by CCRT significantly improved PFS (HR=0.68, 95\% CI 0.56 $0.81, P<0.001)$ compared versus CCRT with or without $A C$, and no heterogeneity was observed $\left(\mathrm{I}^{2}=0.0 \%, P=0.975\right)$. NACT was associated with a significant improvement in overall survival ( $\mathrm{HR}=0.64,95 \% \mathrm{CI} 0.49-0.84, P=0.001 ; \mathrm{I}^{2}=0.0 \%, P=0.467$ ) and distant failure-free survival ( $\mathrm{HR}=0.72,95 \% \mathrm{CI} 0.53-0.97, P=0.031 ; \mathrm{I}^{2}=0.0 \%$, $P=0.744$ ). No significant benefit was shown by NACT for locoregional control. NACT with CCRT increased risks of grade 3 - 4 anemia, thrombocytopenia, leukopenia, and fatigue, compared versus CCRT with or without AC.

Conclusions: Our meta-analysis confirmed that the addition of NACT to CCRT significantly improved PFS and OS versus CCRT with or without AC for locoregionally advanced nasopharyngeal carcinoma. These results may alter the standard of care - CCRT with or without AC, for locoregionally advanced nasopharyngeal carcinoma.

\section{INTRODUCTION}

Nasopharyngeal carcinoma displays a marked geographic distribution, with the highest incidence in Southern China. Radiotherapy is the cornerstone of treatment for nasopharyngeal carcinoma. Radiotherapy alone is recommended for patients who present with T1, N0, M0 disease [1]. In more advanced stages (T1, N1-3 and T2-T4, any N lesions), the standard of care is concurrent chemoradiotherapy (CCRT) with or without adjuvant chemotherapy (AC) [1-3].
The National Comprehensive Cancer Network has widespread disagreement on the role of neoadjuvant chemotherapy (NACT) in the treatment of locoregionally advanced nasopharyngeal carcinoma [1]. A recent metaanalysis demonstrated that NACT followed by CCRT or radiotherapy could improve both overall survival (OS) and progression-free survival (PFS) for patients with locoregionally advanced nasopharyngeal carcinoma, compared with CCRT or radiotherapy [4]. However, the benefit could possibly relate to the superiority of NACT followed by radiotherapy over radiotherapy alone. 
Consequently, it remains unclear whether or not the addition of NACT before CCRT is better than the standard approach of CCRT with or without AC. Four metaanalyses directly [5-6] or indirectly [7-8] showed that NACT plus CCRT did not improved OS compared versus CCRT or CCRT with AC in patients with locoregionally advanced nasopharyngeal carcinoma. However, the largest pair-wise comparison included only 4 trials with a total of 798 patients [6], so these meta-analyses were underpowered by a small sample size [5-8]. In addition, the primary endpoint of all these meta-analyses was OS [5-8]. The longest median follow-up in the included trials was 4.6 years [9], whereas the 5-year survival rate was about $70 \%$ for the advanced disease $[1,10]$. As a result, the duration of follow-up might not have been long enough to determine the effect of NACT on OS. It is hence unclear whether NACT followed by CCRT is better than the standard of care - CCRT with or without AC, for patients with locoregionally advanced nasopharyngeal carcinoma.

The gold standard endpoint for clinical trials of nasopharyngeal carcinoma was OS [11]. However, with augmented applications of secondary and tertiary treatments for nasopharyngeal carcinoma, PFS can be considered as a better primary endpoint than OS [12]. Moreover, PFS appears concordant with OS in trials of combined chemotherapy and radiotherapy for nasopharyngeal carcinoma [11]. In addition to the trials included in the previous meta-analyses, additional randomized studies on the benefit of NACT plus CCRT compared versus CCRT with or without AC in nasopharyngeal carcinoma have been available [1317]. To determine the effect of NACT before CCRT for locoregionally advanced nasopharyngeal carcinoma, we performed this meta-analysis with PFS as the primary endpoint.

\section{RESULTS}

\section{Trials}

The initial search using four English databases (ClinicalTrials.gov, PubMed, EMBASE, and Web of Science) yielded 2130 publications dated until November 13, 2015. Of these, 2106 publications were irrelevant by title or abstract reading. After full text reading, 6 trials were identified to meet the inclusion criteria $[9,13-14,18-20]$. We also found 9 randomized trials in the Chinese literature [15-17, 21-26]. (Figure 1) As a result, a total of 15 randomized trials were identified. Two trials $[13,16]$ included 3 and 6 intervention arms, respectively. For each trial, we combined the interventions into one treatment arm to compare to one control arm, according to the recommendation proposed by the Cochrane Handbook for Systematic Reviews of Interventions [27].

\section{Assessing risk of bias in included trials}

The assessments of risk of bias for each individual trial were described in Supplementary Figure S1. 6 trials were graded as high risk of bias, and were excluded from this meta-analysis [21-26]. The characteristics of these trials were listed in Supplementary Table S1. The other 9 trials were graded as unclear risk of bias, and were eligible for a meta-analysis $[9,13-20]$. The characteristics of these trials were listed in Table 1.

\section{Progression-free survival}

The median follow-up ranged from 18.6 to 55 months. One trial was from non-endemic region (Greece) [9], and the others were from endemic regions [13-20]. Seven trials (1383 patients) investigated NACT plus CCRT versus CCRT alone [9, 14-19]. Two trials (832 patients) investigated NACT plus CCRT versus CCRT plus AC $[13,20]$.

The meta-analysis of PFS was based on 9 trials with 2215 patients [9, 13-20]. The addition of NACT improved PFS (Hazard ratio $[\mathrm{HR}]=0.68,95 \%$ confidence interval [CI] $0.56-0.81, P<0.001$; Figure 2). No heterogeneity was observed among trials $\left(P=0.975, \mathrm{I}^{2}\right.$ $=0.0 \%$ ), confirming the appropriateness of pooling the data. The leave-1-out sensitivity analysis showed that no single trial exerted a significant influence on this result, indicating that the result was reliable. Subgroup analyses were also conducted in order to check whether features of the included trials affected the result of this metaanalysis (Table 2). The association between NACT and an improved PFS was maintained regardless of duration of follow-up, sample size, CCRT timing, radiotherapy technique, NACT regimen, method of data extraction, and with or without AC. The HRs ranged from 0.64 to 0.72 , and there was no evidence of heterogeneity $\left(\mathrm{I}^{2}=0.0 \%\right)$ in all subgroups, suggesting a small variability in the effect of NACT across different inclusion criteria for trials. No significant differences in treatment effect were found across subgroups ( $P$-value for interaction $>0.05$; Table 2$)$.

\section{Overall survival}

Two trials (510 patients), with a median follow-up period of less than 2 years [14, 20], were excluded because they did not fulfill our eligibility criteria. The metaanalysis included 7 trials with 1705 patients [9, 13, 1519]. Overall, NACT before CCRT provided a significant benefit in OS compared versus CCRT with or without AC $(\mathrm{HR}=0.64,95 \%$ CI $0.49-0.84, P=0.001$; Figure 3$)$. There was no evidence of heterogeneity between trials $\left(\mathrm{I}^{2}=0.0 \%, P=0.467\right)$. The sensitivity analysis demonstrated that no single trial exerted a significant influence on the overall result. Table 3 shows the results 
of the subgroup analyses. We identified possible evidence of heterogeneity in subgroup analyses $(n \geq 2)$ when the trials were divided based on NACT regimen (two drugs versus three drugs). Notably, any subgroup with more than 800 patients showed a statistically significant association, whereas no association was shown in only subgroups with fewer than 600 patients. Therefore, the negative results

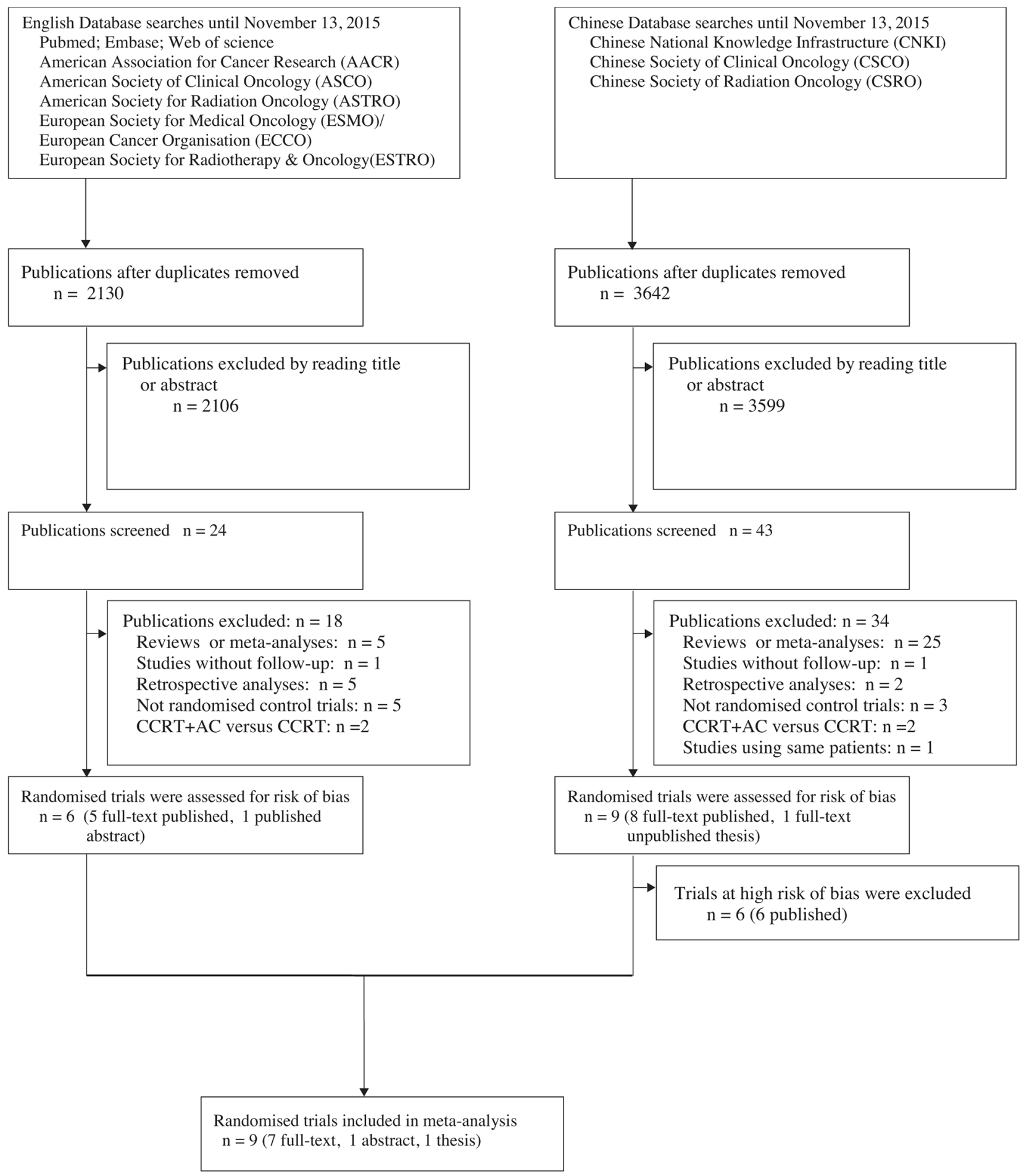

in these subgroups might be attributable to a lack of statistical power (a small sample size) to detect the effect size. No significant interaction was observed between subgroups $(P$-value for interaction $>0.05$; Table 3$)$. The strongest interaction was between NACT regimen and OS: two-drug NACT was more efficient than three-drug NACT

(ratio of $\mathrm{HR}=0.56,95 \%$ CI $0.27-1.15, P=0.119$ ).

American Society for Radiation Oncology (ASTRO)

European Society for Medical Oncology (ESMO)

European Cancer Organisation (ECCO)

European Society for Radiotherapy \& Oncology(ESTRO) ications excluded by reading title astract
Chinese Database searches until November 13,2015

inese National Knowledge Infrastructure (CNKI)

Chinese Society of Clinical Oncology (CSCO)

ublications after duplicates removed

ublications excluded by reading title abtract

Reviews or meta-analyses: $\mathrm{n}=25$

sithout follow-up. $n=1$

Not randomised control trials: $\mathrm{n}=3$

CCRT+AC versus CCRT: $n=2$

Cing same patients: $\mathrm{n}=1$

andomised trials were assessed for risk of bias $=6(6$ published $)$

Figure 1: Flow chart showing inclusion and exclusion of trials. 
Table 1: Description of trials included in the meta-analysis

\begin{tabular}{|c|c|c|c|c|c|c|c|c|c|}
\hline $\begin{array}{l}\text { First author, Year, } \\
\text { (reference) }\end{array}$ & $\begin{array}{c}\text { Race } \\
\text { (Region) }\end{array}$ & $\begin{array}{c}\text { Clinical } \\
\text { stage (TNM } \\
\text { classification) }\end{array}$ & $\begin{array}{c}\text { Histology } \\
\text { (WHO } \\
\text { classification) }\end{array}$ & Radiotherapy & $\begin{array}{c}\text { Concurrent } \\
\text { chemoradiotherapy }\end{array}$ & $\begin{array}{c}\text { Induction } \\
\text { chemotherapy }\end{array}$ & $\begin{array}{c}\text { Adjuvant } \\
\text { chemotherapy }\end{array}$ & $\begin{array}{c}\text { Patients } \\
\text { randomized } \\
\text { (treatment/ } \\
\text { control) }\end{array}$ & $\begin{array}{l}\text { Median } \\
\text { follow-up } \\
\text { (month) }\end{array}$ \\
\hline Fountzilas et al., 2012, [9] & $\begin{array}{c}\text { Greek } \\
\text { (Greece) }\end{array}$ & $\begin{array}{l}\text { AJCC/UICC } \\
\text { 6th edition } \\
\text { IIB-IVB }\end{array}$ & $1-3$ & $\begin{array}{l}\text { (79 patients) 3D-CRT } \\
\text { and ( } 62 \text { patients) } \\
\text { 2D-CRT: } 2.0 \mathrm{~Gy} / \mathrm{F} \times 5 \mathrm{~F} / \\
\text { wk; primary site } 66-70 \\
\text { Gy; positive nodes } \\
66-70 \mathrm{~Gy} \text {; pharyngeal } \\
\text { extension and residual } \\
\text { nodes } 50 \text { Gy. }\end{array}$ & $\begin{array}{l}\text { Cisplatin } 40 \mathrm{mg} / \mathrm{m}^{2} \\
\mathrm{~d} 1, \mathrm{q} 1 \mathrm{wk} \times 8\end{array}$ & $\begin{array}{l}\text { Cisplatin } 75 \\
\mathrm{mg} / \mathrm{m}^{2} \mathrm{~d} 1 ; \\
\text { Epirubicin } \\
75 \mathrm{mg} / \mathrm{m}^{2} \mathrm{~d} 1 ; \\
\text { Paclitaxel } 175 \\
\mathrm{mg} / \mathrm{m}^{2} \mathrm{~d} 1 ; \\
\mathrm{q} 3 \mathrm{wks} \times 3\end{array}$ & & $72 / 69$ & $\begin{array}{c}55 \\
(0.5-76.2)\end{array}$ \\
\hline Tan et al. 2015, [18] & $\begin{array}{l}\text { Singaporean } \\
\text { (Singapore) }\end{array}$ & $\begin{array}{l}\text { UICC/AJCC } \\
\text { 5th edition } \\
\text { T3-4NxM0 or } \\
\text { TxN2-3M0 }\end{array}$ & 2,3 & $\begin{array}{l}\text { (168 patients) } \\
\text { IMRT: GTVnx: } \\
\text { 69.96Gy/2.12Gy/33F; } \\
\text { GTVnd: } \\
\text { 69.96Gy/2.12Gy/33F; } \\
\text { CTV1: } \\
\text { 60Gy/1.82Gy/33F. } \\
\text { (4 patients) 2D-CRT: } \\
\text { primary site } \\
\text { 70Gy/2Gy/35F; positive } \\
\text { nodes } 70 \mathrm{~Gy} / 2 \mathrm{~Gy} / 35 \mathrm{~F} ; \\
\text { pharyngeal extension } \\
\text { and residual nodes } \\
\text { 60Gy/2Gy/30F. }\end{array}$ & $\begin{array}{l}\text { Cisplatin } 40 \mathrm{mg} / \mathrm{m}^{2} \\
\mathrm{~d} 1 \text {, q1wk } \times 8\end{array}$ & $\begin{array}{l}\text { Paclitaxel } \\
70 \mathrm{mg} / \mathrm{m}^{2} \mathrm{~d} 1, \\
\mathrm{~d} 8 ; \text { Carboplatin } \\
\text { AUC }=2.5 \\
\mathrm{~d} 1, \mathrm{~d} 8 ; \\
\text { Gemcitabine } \\
1000 \mathrm{mg} / \mathrm{m}^{2} \mathrm{~d} 1, \\
\mathrm{~d} 8 ; \mathrm{q} 3 \mathrm{wks} \times 3\end{array}$ & & $86 / 86$ & $\begin{array}{c}40.8 \\
(13.2-100.8)\end{array}$ \\
\hline Ma et al. 2014, [14] & $\begin{array}{l}\text { Chinese } \\
\text { (Mainland } \\
\text { China) }\end{array}$ & $\begin{array}{l}\text { UICC/AJCC } \\
\text { 7th edition } \\
\text { III-IVB } \\
\text { (except T3- } \\
\text { 4N0) }\end{array}$ & 2,3 & $\begin{array}{l}\text { IMRT (radical } \\
\text { radiotherapy) }\end{array}$ & $\begin{array}{l}\text { Cisplatin } 100 \mathrm{mg} / \mathrm{m}^{2} \\
\mathrm{~d} 1, \mathrm{q} 3 \mathrm{wks} \times 3\end{array}$ & $\begin{array}{l}\text { Docetaxel } \\
60 \mathrm{mg} / \mathrm{m}^{2} \\
\mathrm{~d} 1 ; \text { Cisplatin } \\
60 \mathrm{mg} / \mathrm{m}^{2} \mathrm{~d} 1 ; \\
\text { Fluorouracil } \\
600 \mathrm{mg} / \mathrm{m}^{2} \mathrm{~d} 1- \\
5 ; \mathrm{q} 3 \mathrm{wks} \times 3\end{array}$ & & $241 / 239$ & $\begin{array}{c}18.6 \\
(0.8-34)\end{array}$ \\
\hline Hui et al. 2009, [19] & $\begin{array}{l}\text { Chinese } \\
\text { (Hong } \\
\text { Kong) }\end{array}$ & $\begin{array}{l}\text { UICC/AJCC } \\
\text { 5th edition } \\
\text { III-IVB }\end{array}$ & NR & $\begin{array}{l}\text { (17 patients) IMRT and } \\
\text { (48 patients) 2D-CRT: } \\
2 \mathrm{~Gy} / \mathrm{F} \times 5 \mathrm{~F} / \text { wk; total } 66 \\
\text { Gy; residual boost of } \\
7.5 \mathrm{~Gy} \text {. }\end{array}$ & $\begin{array}{l}\text { Cisplatin } 40 \mathrm{mg} / \mathrm{m}^{2} \\
\mathrm{~d} 1, \mathrm{q} 1 \mathrm{wk} \times 8\end{array}$ & $\begin{array}{l}\text { Docetaxel } 75 \\
\mathrm{mg} / \mathrm{m}^{2} \mathrm{~d} 1 \\
\text { Cisplatin } 75 \\
\mathrm{mg} / \mathrm{m}^{2} \mathrm{~d} 1 \\
\mathrm{q} 3 \mathrm{wk} \times 2\end{array}$ & & $34 / 31$ & 51.6 \\
\hline Huang et al. 2012, [17] & $\begin{array}{l}\text { Chinese } \\
\text { Mainland } \\
\text { (China) }\end{array}$ & $\begin{array}{l}\text { AJCC/UICC } \\
\text { 6th edition } \\
\text { III-IVB }\end{array}$ & 2,3 & $\begin{array}{l}\text { 2D-CRT: } 2.0 \mathrm{~Gy} / \mathrm{F} \times 5 \mathrm{~F} / \\
\text { wk; primary site } 65-78 \\
\text { Gy; positive nodes } \\
60-70 \mathrm{~Gy} \text {; pharyngeal } \\
\text { extension and residual } \\
\text { nodes } 50-54 \mathrm{~Gy} \text {. }\end{array}$ & $\begin{array}{l}\text { Caboplatin AUC }=6 \\
\text { d7, d28, d49 }\end{array}$ & $\begin{array}{l}\text { Caboplatin } \\
\text { AUC }=6 \mathrm{~d} 1 \text {; } \\
\text { Fluorouracil } \\
750 \mathrm{mg} / \mathrm{m}^{2}, \mathrm{~d} 1- \\
5 ; \mathrm{q} 3 \mathrm{wks} \times 2\end{array}$ & & $100 / 100$ & 46.8 \\
\hline Gao et al. 2013, [15] & $\begin{array}{c}\text { Chinese } \\
\text { (Mainland } \\
\text { China) }\end{array}$ & $\begin{array}{l}1992 \text { Fuzhou } \\
\text { stage T3- } \\
\text { 4N2-3M0 }\end{array}$ & 2,3 & $\begin{array}{l}\text { 2D-CRT: } 2.0 \mathrm{~Gy} / \mathrm{F} \times 5 \mathrm{~F} / \\
\text { wk; primary site } 70-74 \\
\text { Gy; positive nodes } \\
66-70 \mathrm{~Gy} \text {; pharyngeal } \\
\text { extension and residual } \\
\text { nodes } 50 \mathrm{~Gy} \text {. }\end{array}$ & $\begin{array}{l}\text { Cisplatin } 40 \mathrm{mg} / \mathrm{m}^{2} \\
\mathrm{~d} 1, \mathrm{q} 1 \mathrm{wk} \times 7\end{array}$ & $\begin{array}{l}\text { Cisplatin } \\
30 \mathrm{mg} / \mathrm{m}^{2} \mathrm{~d} 1-3 \\
\text { Fluorouracil } \\
450 \mathrm{mg} / \mathrm{m}^{2} \mathrm{~d} 1- \\
3 ; \mathrm{q} 3 \mathrm{wks} \times 2\end{array}$ & & $57 / 55$ & $42(>24)$ \\
\hline Sun, 2009, $[16]^{\text {a }}$ & $\begin{array}{l}\text { Chinese } \\
\text { (Mainland } \\
\text { China) }\end{array}$ & $\begin{array}{l}1992 \text { Fuzhou } \\
\text { stage III-IVA }\end{array}$ & 2,3 & $\begin{array}{l}\text { (156 patients) IMRT: } \\
\text { 5F/wk; GTVnx: } \\
\text { 68Gy/30F; GTVnd: } \\
\text { 60-66Gy/30F; CTVl: } \\
\text { 60Gy/30F; CTV2: } \\
\text { 54Gy/30F. (57 patients) } \\
\text { 2D-CRT: 2.0Gy/F×5F/ } \\
\text { wk; primary site } 70 \\
\text { Gy; positive nodes } \\
\text { 66-70 Gy; pharyngeal } \\
\text { extension and residual } \\
\text { nodes } 50 \text { Gy. }\end{array}$ & $\begin{array}{l}\text { Cisplatin } 80 \mathrm{mg} / \mathrm{m}^{2} \\
\mathrm{~d} 1, \mathrm{q} 3 \mathrm{wks} \times 2\end{array}$ & $\begin{array}{l}\text { Group } 1 \\
\text { ( } 76 \text { patients): } \\
\text { Cisplatin } \\
80 \mathrm{mg} / \mathrm{m}^{2} \mathrm{~d} 1 ; \\
\text { Fluorouracil } \\
1.5 \mathrm{~g} / \mathrm{m}^{2} \mathrm{~d} 1-2 \text {; } \\
\mathrm{q} 3 \mathrm{wks} \times 2 \\
\text { Group } 2 \text { (66 } \\
\text { patients) : } \\
\text { Fluorouracil } \\
1.5 \mathrm{~g} / \mathrm{m}^{2} \mathrm{~d} 1-2 ; \\
\text { Carboplatin } \\
\text { AUC }=6 ; \\
\mathrm{q} 3 \mathrm{wks} \times 2\end{array}$ & & $142 / 71$ & $\begin{array}{c}26.3 \\
(2.5-44.7)\end{array}$ \\
\hline Lee et al. 2015, [13] ${ }^{\mathrm{b}}$ & $\begin{array}{l}\text { Chinese } \\
\text { (Hong } \\
\text { Kong and } \\
\text { Mainland } \\
\text { China) }\end{array}$ & $\begin{array}{l}\text { AJCC/UICC } \\
\text { 6th edition } \\
\text { III-IVB }\end{array}$ & 2,3 & $\begin{array}{l}\text { IMRT }^{5} 2.0 \mathrm{~Gy} / \mathrm{F} \times 5 \mathrm{~F} / \\
\text { wk or } 2.0 \mathrm{~Gy} / \mathrm{F} \times 6 \mathrm{~F} / \\
\text { wk; gross tumor target } \\
70 \mathrm{~Gy} \text {; positive nodes } \\
<70 \mathrm{~Gy} \text {; pharyngeal } \\
\text { extension and residual } \\
\text { nodes } 50 \mathrm{~Gy} \text {. }\end{array}$ & $\begin{array}{l}\text { Cisplatin } 100 \mathrm{mg} / \\
\mathrm{m} 2 \mathrm{~d} 1, \mathrm{q} 3 \mathrm{wks} \times 2 / 3\end{array}$ & $\begin{array}{l}\text { Cisplatin } 100 \\
\mathrm{mg} / \mathrm{m} 2 \mathrm{~d} 1, \\
\text { Fluorouracil } \\
1 \mathrm{~g} / \mathrm{m} 2 \mathrm{~d} 1-5 \text {, } \\
\mathrm{q} 3 \mathrm{wks} \times 2 / 3 \text {; or } \\
\text { Cisplatin } 100 \\
\mathrm{mg} / \mathrm{m} 2 \mathrm{~d} 1 \text {, } \\
\text { capecitabine } \\
2 \mathrm{~g} / \mathrm{m} 2 \mathrm{~d} 1-14 \text {, } \\
\mathrm{q} 3 \mathrm{wks} \times 2 / 3\end{array}$ & $\begin{array}{l}\text { Cisplatin } 80 \\
\mathrm{mg} / \mathrm{m} 2 \mathrm{~d} 1 \text {, } \\
\text { Fluorouracil } \\
1 \mathrm{~g} / \mathrm{m} 2 \mathrm{~d} 1-4, \\
\text { q4wks } \times 3\end{array}$ & $538 / 264$ & $\begin{array}{c}39.6 \\
(1.2-85.2)\end{array}$ \\
\hline
\end{tabular}




\begin{tabular}{|c|c|c|c|c|c|c|c|c|c|}
\hline $\begin{array}{l}\text { First author, Year, } \\
\text { (reference) }\end{array}$ & $\begin{array}{c}\text { Race } \\
\text { (Region) }\end{array}$ & $\begin{array}{c}\text { Clinical } \\
\text { stage (TNM } \\
\text { classification) }\end{array}$ & $\begin{array}{c}\text { Histology } \\
\text { (WHO } \\
\text { classification) }\end{array}$ & Radiotherapy & $\begin{array}{c}\text { Concurrent } \\
\text { chemoradiotherapy }\end{array}$ & $\begin{array}{c}\text { Induction } \\
\text { chemotherapy }\end{array}$ & $\begin{array}{c}\text { Adjuvant } \\
\text { chemotherapy }\end{array}$ & $\begin{array}{c}\text { Patients } \\
\text { randomized } \\
\text { (treatment/ } \\
\text { control) }\end{array}$ & $\begin{array}{c}\text { Median } \\
\text { follow-up } \\
\text { (month) }\end{array}$ \\
\hline Ruste et al. 2011, [20] & $\begin{array}{l}\text { Philippinese } \\
\text { (Philippines) }\end{array}$ & III-IVB & 2,3 & $\begin{array}{l}\text { 2D-CRT: } 2.0 \mathrm{~Gy} / \mathrm{F} \times 5 \mathrm{~F} / \\
\text { wk; primary site } 70 \mathrm{~Gy} \text {, } \\
\text { N0 disease } 50 \mathrm{~Gy}, \\
\text { nodes }<2 \mathrm{~cm} 66 \mathrm{~Gy}, \\
\text { nodes greater than } 2 \mathrm{~cm} \\
70 \mathrm{~Gy} \text {. }\end{array}$ & $\begin{array}{l}\text { Cisplatin } 25 \mathrm{mg} / \mathrm{m} 2 \\
\mathrm{~d} 1-4, \mathrm{q} 3 \mathrm{wks} \times 3\end{array}$ & $\begin{array}{l}\text { Cisplatin } 20 \\
\mathrm{mg} / \mathrm{m} 2 \mathrm{~d} 1-4 \text {, } \\
\mathrm{q} 4 \mathrm{wks} \times 3 ; \\
5 \text {-Fluorouracil } \\
1000 \mathrm{mg} / \mathrm{m} 2 \\
\mathrm{~d} 1-4 ; \mathrm{q} 4 \mathrm{wks} \times 3\end{array}$ & $\begin{array}{l}\text { Cisplatin } 20 \\
\mathrm{mg} / \mathrm{m} 2 \mathrm{~d} 1-4 ; \\
5 \text {-Fluorouracil } \\
1000 \mathrm{mg} / \\
\mathrm{m} 2 \mathrm{~d} 1-4 ; \\
\mathrm{q} 4 \mathrm{wks} \times 3\end{array}$ & $14 / 16$ & $\begin{array}{c}19 \\
(8-30)\end{array}$ \\
\hline
\end{tabular}

a Patients were allocated to 6 arms: arm 1A (conventional fractionation radiotherapy, adjuvant chemotherapy using Cisplatin plus 5-Fluorouracil), arm 2A (conventional fractionation radiotherapy, neoadjuvant chemotherapy using Cisplatin plus 5-Fluorouracil), arm 3A (conventional fractionation radiotherapy, neoadjuvant chemotherapy using Cisplatin plus Capecitabine), arm 1B (accelerated fractionation radiotherapy, adjuvant chemotherapy using Cisplatin plus 5-Fluorouracil), arm 2B (accelerated fractionation radiotherapy, neoadjuvant chemotherapy using Cisplatin plus 5-Fluorouracil), arm 3B (accelerated fractionation radiotherapy, neoadjuvant chemotherapy using Cisplatin plus Capecitabine). In this meta-analysis, we combined arm 2A, $\operatorname{arm} 3 \mathrm{~A}$, arm 2B, and arm 3B together as a treatment group, and combined arm 1A and arm 1B together as a control group. The influence of the radiotherapy fractionation (conventional and accelerated) on the present meta-analysis of progression-free survival and overall survival was shown in Supplementary Table S2.

${ }^{b}$ Patients were divided into three study groups, comparing concurrent chemoradiotherapy (control group) with two different NACT plus CCRT treatments (treatment group). For the purpose of the present meta-analysis, the control group was directly used, and the two treatment groups were pooled together.

2D-CRT, Two-dimensional conformal radiation therapy; 3D-CRT, Three-dimensional conformal radiation therapy; AJCC, American Joint Committee on Cancer; AUC, area under the curve; CTV, clinical target volume; F, fraction; GTV, Gross tumor volume; IMRT, intensity-modulated radiotherapy; TNM, Tumour Nodes Metastasis; UICC, International Union Against Cancer; WHO, World Health Organization; d, day; q1wk, every 1 week; q3wk, every 3 weeks; wk, week.

c $95 \%$ patients were treated with IMRT.

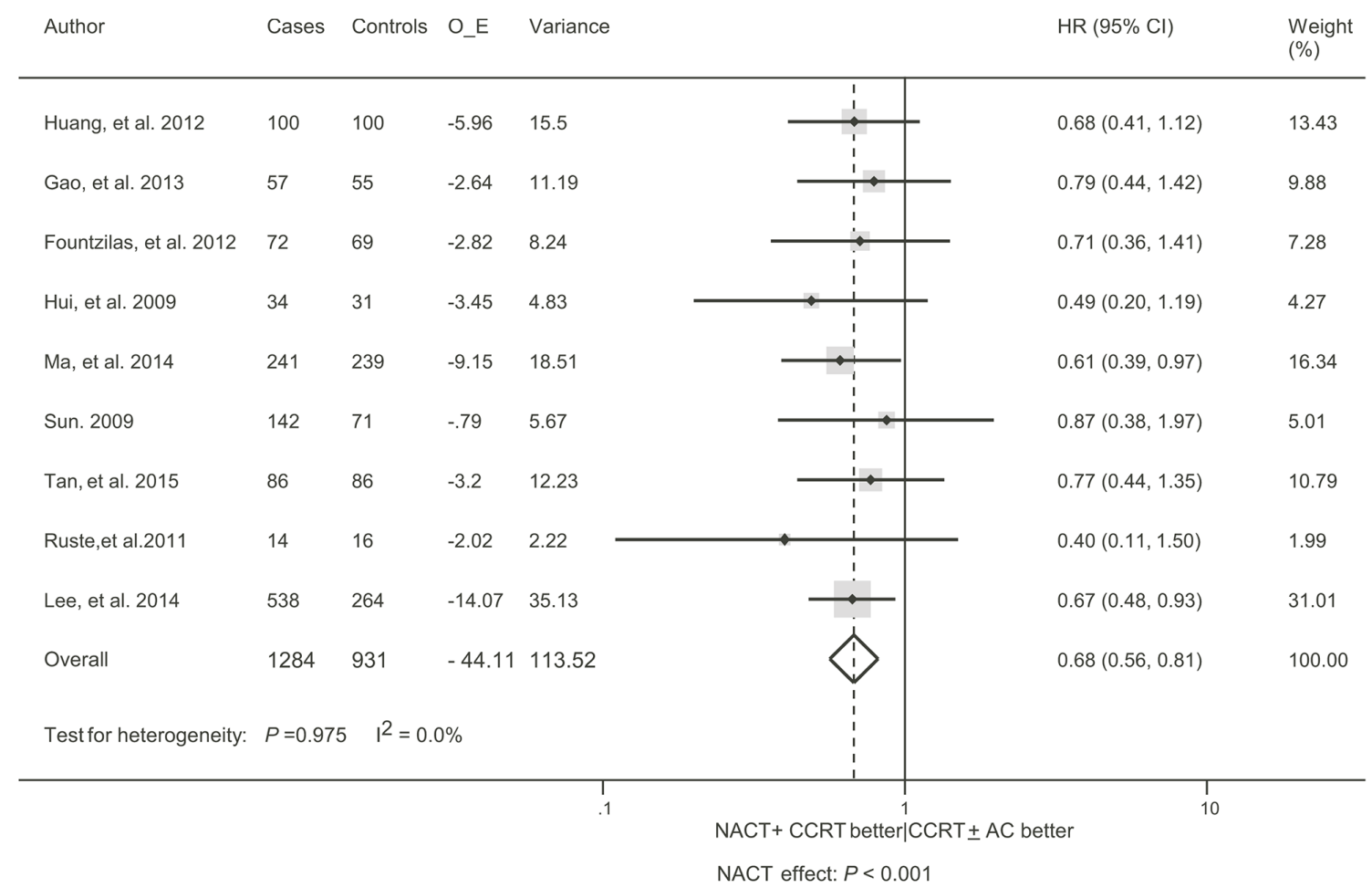

Figure 2: Forest plot for the hazard ratio of progression-free survival with neoadjuvant chemotherapy followed by concurrent chemoradiotherapy versus concurrent chemoradiotherapy with or without adjuvant chemotherapy for locoregionally advanced nasopharyngeal carcinoma. AC, adjuvant chemotherapy; CCRT, concurrent chemoradiotherapy; CI, confidence interval; HR, hazard ratio; NACT, neoadjuvant chemotherapy; O-E, observed minus expected events.

\section{Distant failure-free survival and locoregional failure}

As for distant failure-free survival (DFFS), the meta-analysis included 5 trials with 1177 patients [14-18]. A significant benefit in favor of the addition of NACT was found without evidence of heterogeneity $(\mathrm{HR}=0.72,95 \%$ CI, $0.53-0.97, P=0.031 ; I^{2}=0.0 \%, P=0.744$; Figure 4).
The sensitivity analysis demonstrated the trial by Ma et al. [14] exerting a significant influence on the overall result. The HR was non-significant $(\mathrm{HR}=0.82,95 \% \mathrm{CI}$, $\left.0.57-1.16, P=0.255 ; \mathrm{I}^{2}=0.0 \%, P=0.999\right)$ when this trial was excluded. Data regarding the absolute number of locoregional failure were available in 4 trials with 591 patients [9, 16, 18-19]. There was no benefit in favor of the addition of NACT, without evidence of heterogeneity 
Table 2: Subgroup analyses for the treatment effect on progression-free survival of neoadjuvant chemotherapy followed by concurrent chemoradiotherapy versus concurrent chemoradiotherapy with or without adjuvant chemotherapy for locoregionally advanced nasopharyngeal carcinoma

\begin{tabular}{|c|c|c|c|c|c|c|c|c|}
\hline \multirow[t]{2}{*}{ Factors } & \multirow[t]{2}{*}{ Subgroups } & \multicolumn{2}{|c|}{ Availability } & \multicolumn{2}{|c|}{ Effect } & \multicolumn{2}{|c|}{ Heterogeneity } & Interaction \\
\hline & & $\begin{array}{c}\text { Trials } \\
(N)\end{array}$ & $\begin{array}{c}\text { Patients } \\
(N)\end{array}$ & $\begin{array}{c}\text { HR } \\
(95 \% \text { CI })\end{array}$ & $P$ value & $\mathbf{I}^{2}$ & $P$ value & $P$ value \\
\hline
\end{tabular}

Duration of follow-up

$\begin{array}{lccccccc}>36 \text { months } & 6 & 1492 & 0.69(0.56-0.85) & 0.001 & 0.0 & 0.966 & 0.748 \\ <36 \text { months } & 3 & 723 & 0.64(0.43-0.93) & 0.020 & 0.0 & 0.585 & \end{array}$

Sample size

$\begin{array}{cccccccc}<150 \text { patients } & 4 & 348 & 0.66(0.45-0.97) & 0.033 & 0.0 & 0.944 & 0.915 \\ >150 \text { patients } & 5 & 1867 & 0.68(0.55-0.84) & 0.000 & 0.0 & 0.706 & \end{array}$

CCRT timing a

$\begin{array}{llllllll}\text { q1wk } & 4 & 490 & 0.72(0.52-0.97) & 0.045 & 0.0 & 0.833 & 0.669 \\ \text { q3wk } & 5 & 1725 & 0.66(0.53-0.83) & 0.000 & 0.0 & 0.889 & \end{array}$

Method of data extraction ${ }^{b}$

$\begin{array}{lccccccc}\begin{array}{l}\text { Directly } \\ \text { reported }\end{array} & 5 & 1660 & 0.66(0.53-0.82) & 0.000 & 0.0 & 0.927 & 0.791 \\ \begin{array}{l}\text { Indirect } \\ \text { method }\end{array} & 4 & 555 & 0.72(0.51-0.98) & 0.048 & 0.0 & 0.771 & \end{array}$

Radiotherapy technique ${ }^{\mathrm{c}}$

$\begin{array}{lccccccc}\begin{array}{l}\text { Conventional } \\ \text { radiotherapy }\end{array} & 4 & 407 & 0.66(0.47-0.92) & 0.015 & 0.0 & 0.713 & 0.872 \\ \text { IMRT/3DCRT } & 5 & 1808 & 0.69(0.55-0.85) & 0.001 & 0.0 & 0.943\end{array}$

$\mathrm{IC}$ regimen

$\begin{array}{lccccccc}\text { Two drugs } & 6 & 1422 & 0.68(0.54-0.85) & 0.001 & 0.0 & 0.882 & 0.952 \\ \begin{array}{l}\text { Three drugs } \\ \begin{array}{l}\text { Taxol- } \\ \text { included }\end{array}\end{array} & 3 & 793 & 0.68(0.50-0.93) & 0.015 & 0.0 & 0.810 & \\ \begin{array}{l}\text { Non-taxol- } \\ \text { included }\end{array} & 5 & 1357 & 0.69(0.55-0.88) & 0.002 & 0.0 & 0.832 & 0.876 \\ \end{array}$

$\mathrm{AC}$

$\begin{array}{lccccccc}\text { With } & 7 & 1383 & 0.65(0.47-0.90) & 0.008 & 0.0 & 0.959 & 0.812 \\ \text { Without } & 2 & 832 & 0.70(0.55-0.87) & 0.005 & 0.0 & 0.453 & \end{array}$

Data source

\begin{tabular}{lccccccc} 
Published & 7 & 1522 & $0.68(0.55-0.84)$ & $<0.001$ & 0.0 & 0.954 & 0.904 \\
Unpublished & 2 & 693 & $0.66(0.45-0.99)$ & 0.043 & 0.0 & 0.459 & \\
\hline
\end{tabular}

a Including one trial in which chemotherapy was administered every 4 weeks [20].

${ }^{\mathrm{b}} \mathrm{HR}$ and its $95 \%$ CI were directly reported or indirectly calculated according to the method by Parmar et al. [46] in a trial.

${ }^{\mathrm{c}}$ Radiotherapy technique was classified as conventional radiotherapy or IMRT/3D-CRT based on the radiotherapy applied for at least $70 \%$ of the study population.

3D-CRT, Three-dimensional conformal radiation therapy; AC, adjuvant chemotherapy; CCRT, concurrent chemoradiotherapy; CI, confidence interval; DFFS, distant failure-free survival; HR, hazard ratio; NACT, neoadjuvant chemotherapy; IMRT, intensity-modulated radiotherapy; q1wk, every 1 week; q3wk, every 3 weeks. 
(odds ratio $[\mathrm{OR}]=1.31,95 \% \mathrm{CI}, 0.83-2.07, P=0.254$; $\mathrm{I}^{2}=0.0 \%, P=0.602$; Figure 5 ). The sensitivity analysis showed that no single trial exerted a significant influence on the overall result.

\section{Treatment-related adverse events}

Table 4 described the acute and late grade $3-4$ adverse events that were observed in these trials. The most common grade $3-4$ acute adverse events were leukopaenia (22.5\%), mucositis (17.9\%), and esophagitis (15.4\%). 20 acute adverse events, which were reported by more than 2 trials, were meta-analyzed. 7 acute adverse events, including anemia, dermatitis, mucositis, and nausea/vomiting, thrombocytopenia, leucopenia, and nephrotoxicity were reported by more than 5 trials with a sample size of more than 860 . The addition of NACT before CCRT was associated with a significantly increased risk of anemia $(\mathrm{OR}=1.87,95 \%$ CI 1.04-3.34, $\left.P=0.036 ; \mathrm{I}^{2}=7.8 \%, P=0.370\right)$, thrombocytopenia $\left(\mathrm{OR}=3.67,95 \%\right.$ CI 1.85-7.23, $P<0.001 ; \mathrm{I}^{2}=14.6 \%$, $P=0.319)$, leucopenia $(\mathrm{OR}=2.13,95 \%$ CI $1.00-4.57$, $\left.P=0.050 ; \mathrm{I}^{2}=70.8 \%, P=0.004\right)$, and fatigue $(\mathrm{OR}=$ $2.47,95 \%$ CI $1.03-5.94, P=0.043 ; \mathrm{I}^{2}=49.4 \%, P=$ 0.139), compared versus CCRT with or without AC. The incidences of other acute adverse events were comparable between the two arms. There was some evidence of heterogeneity between trials in the acute adverse event analyses. 6 late adverse events, which were mainly related to radiotherapy, were reported by at least 2 trials. The incidences of the late adverse events were not statistically different between the two arms. Because most of these meta-analyses included a small number of samples, a lack of statistical power should be considered.

\section{Publication bias}

Asymmetry in the funnel plot was not observed in the meta-analysis of the effect of NACT on PFS (Supplementary Figure S2). No publication bias was suggested by Begg's rank correlation test $(P=0.999)$ and Egger's linear regression test $(P=0.581)$. Tests of publication bias were not conducted for the other meta-analyses because too few studies were available to make a valid statistical test.

\section{DISCUSSION}

Compared with previous meta-analyses [5-8], this study included more trials (Table 5), and therefore was able to employ rigorous methodology to estimate the effect of NACT on locoregionally advanced nasopharyngeal carcinoma. The results of this meta-analysis suggested that the advantages of NACT followed by CCRT over CCRT with or without

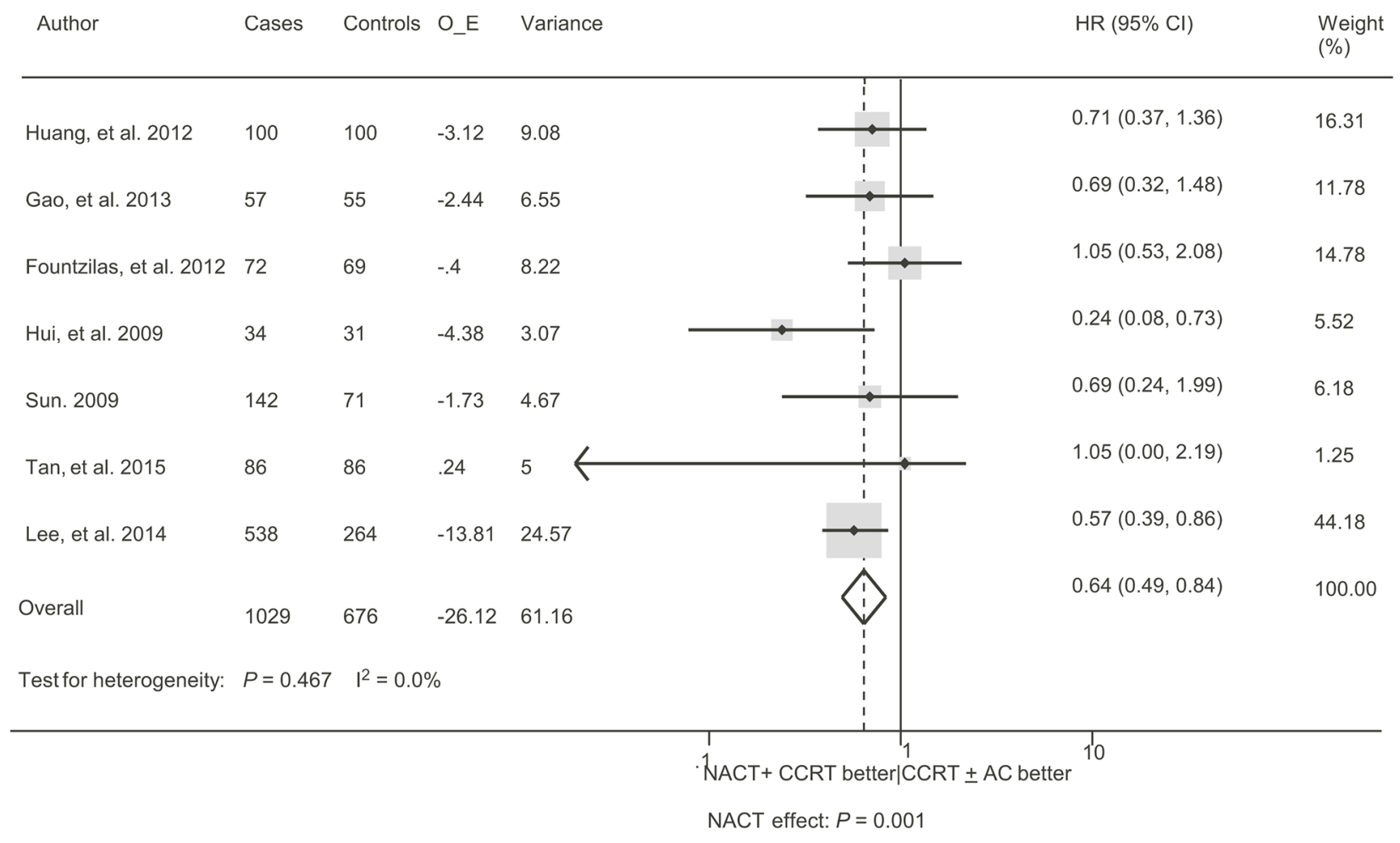

Figure 3: Forest plot for the hazard ratio of overall survival with neoadjuvant chemotherapy followed by concurrent chemoradiotherapy versus concurrent chemoradiotherapy with or without adjuvant chemotherapy for locoregionally advanced nasopharyngeal carcinoma. See Figure 2 for abbreviations. 
Table 3: Subgroup analyses for the treatment effect on overall survival of neoadjuvant chemotherapy followed by concurrent chemoradiotherapy versus concurrent chemoradiotherapy with or without adjuvant chemotherapy for locoregionally advanced nasopharyngeal carcinoma

\begin{tabular}{|c|c|c|c|c|c|c|c|c|}
\hline \multirow[t]{2}{*}{ Factors } & \multirow[t]{2}{*}{ Subgroups } & \multicolumn{2}{|c|}{ Availability } & \multicolumn{2}{|c|}{ Effect } & \multicolumn{2}{|c|}{ Heterogeneity } & Interaction \\
\hline & & $\begin{array}{c}\text { Trials } \\
(N)\end{array}$ & $\begin{array}{l}\text { Patients } \\
(N)\end{array}$ & $\begin{array}{c}\text { HR } \\
(95 \% \mathrm{CI})\end{array}$ & $P$ value & $\mathbf{I}^{2}$ & $P$ value & $P$ value \\
\hline
\end{tabular}

Duration of follow-up

$\begin{array}{llllllll}<48 \text { months } & 4 & 1327 & 0.62(0.47-0.84) & 0.002 & 0.0 & 0.927 & 0.960 \\ >48 \text { months } & 3 & 378 & 0.60(0.19-1.87) & 0.380 & 59.6 & 0.084 & \end{array}$

Radiotherapy technique ${ }^{\mathrm{a}}$

$\begin{array}{lccccccc}\begin{array}{l}\text { Conventional } \\ \text { radiotherapy }\end{array} & 3 & 377 & 0.59(0.38-0.92) & 0.021 & 32.7 & 0.226 & 0.285 \\ \text { IMRT/3DCRT } & 4 & 1328 & 0.67(0.49-0.93) & 0.015 & 0.0 & 0.492 & \end{array}$

Sample size

$\begin{array}{cccccccc}<150 \text { patients } & 3 & 318 & 0.62(0.29-1.32) & 0.216 & 59.0 & 0.087 & 0.999 \\ >150 \text { patients } & 4 & 1387 & 0.62(0.45-0.85) & 0.003 & 0.0 & 0.912 & \end{array}$

CCRT timing ${ }^{b}$

$\begin{array}{lccccccc}\text { q1wk } & 4 & 490 & 0.65(0.34-1.25) & 0.198 & 39.5 & 0.175 & 0.872 \\ \text { q3wk } & 3 & 1215 & 0.61(0.45-0.84) & 0.003 & 0.0 & 0.826 & \end{array}$

Method of data extraction ${ }^{c}$

$\begin{array}{lccccccc}\begin{array}{l}\text { Directly } \\ \text { reported }\end{array} & 4 & 1180 & 0.61(0.44-0.85) & 0.003 & 0.0 & 0.999 & 0.755 \\ \begin{array}{l}\text { Indirect } \\ \text { method }\end{array} & 3 & 525 & 0.70(0.45-1.09) & 0.112 & 44.1 & 0.147 & \end{array}$

IC regimen

\begin{tabular}{|c|c|c|c|c|c|c|c|}
\hline Two drugs & 5 & 1392 & $0.59(0.44-0.78)$ & 0.000 & 0.0 & 0.543 & 0.119 \\
\hline Three drugs & 2 & 313 & $1.05(0.54-2.04)$ & 0.885 & 0.0 & 1.000 & \\
\hline $\begin{array}{l}\text { Taxol- } \\
\text { included }\end{array}$ & 3 & 378 & $0.60(0.20-1.87)$ & 0.380 & 59.6 & 0.084 & 0.962 \\
\hline $\begin{array}{l}\text { Non-taxol- } \\
\text { included }\end{array}$ & 4 & 1327 & $0.62(0.47-0.84)$ & 0.002 & 0.0 & 0.927 & \\
\hline
\end{tabular}

$\mathrm{AC}$

$\begin{array}{lllllccc}\text { With } & 1 & 802 & 0.64(0.49-0.83) & 0.005 & - & - & 0.798 \\ \text { Without } & 6 & 903 & 0.70(0.50-0.98) & 0.040 & 0.0 & 0.420 & \end{array}$

Data source

\begin{tabular}{lccccccc} 
Published & 6 & 1492 & $0.64(0.48-0.87)$ & 0.004 & 10.2 & 0.351 & 0.913 \\
Unpublished & 1 & 213 & $0.69(0.25-1.93)$ & 0.883 & - & - & \\
\hline
\end{tabular}

a Radiotherapy technique was classified as conventional radiotherapy or IMRT/3D-CRT based on the radiotherapy applied for at least $70 \%$ of the study population.

${ }^{\mathrm{b}} \mathrm{HR}$ and its $95 \% \mathrm{CI}$ were directly reported or indirectly calculated according to the method by Parmar et al. [46] in a trial.

c Including one trial in which chemotherapy was administered every 4 weeks[20].

See Table 2 for abbreviations. 


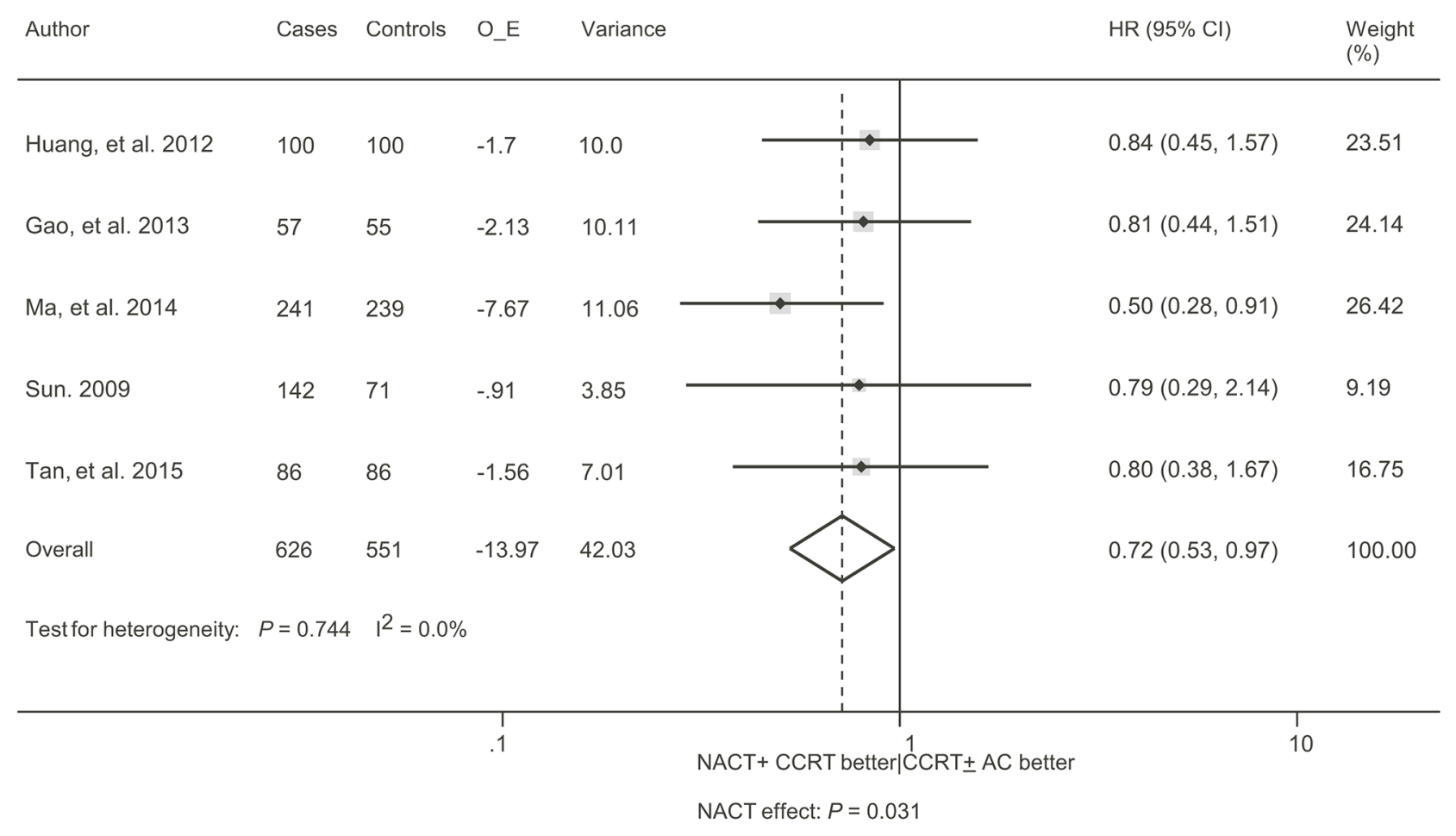

Figure 4: Forest plot for the hazard ratio of distant failure-free survival with neoadjuvant chemotherapy followed by concurrent chemoradiotherapy versus concurrent chemoradiotherapy with or without adjuvant chemotherapy for locoregionally advanced nasopharyngeal carcinoma. See Figure 2 for abbreviations.

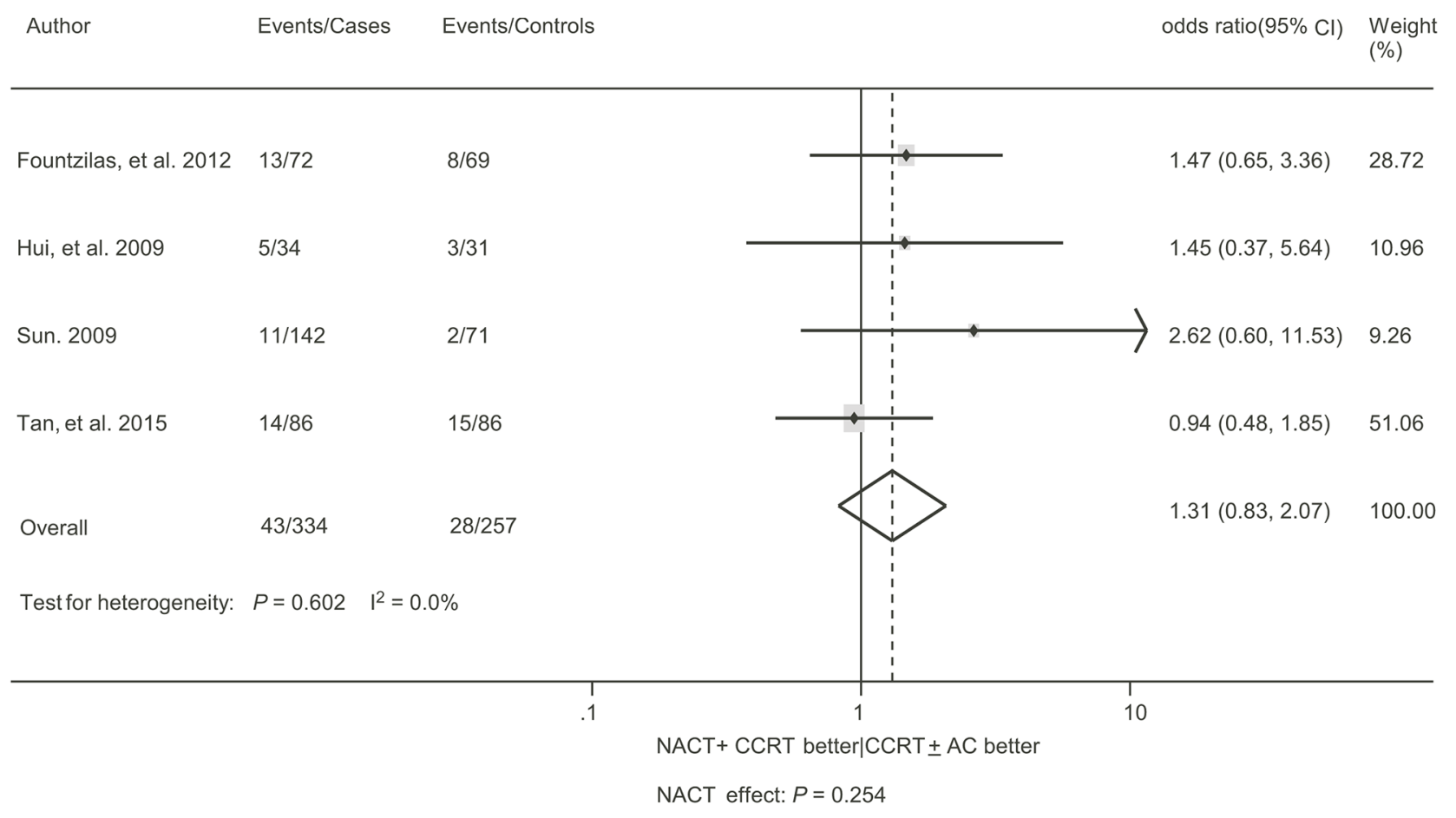

Figure 5: Forest plot of the odds ratio of locoregional failure with neoadjuvant chemotherapy followed by concurrent chemoradiotherapy versus concurrent chemoradiotherapy with or without adjuvant chemotherapy for locoregionally advanced nasopharyngeal carcinoma. See Figure 2 for abbreviations. 
Table 4: Severe adverse events of neoadjuvant chemotherapy followed by concurrent chemoradiotherapy versus concurrent chemoradiotherapy with or without adjuvant chemotherapy for locoregionally advanced nasopharyngeal carcinoma

\begin{tabular}{|c|c|c|c|c|c|c|c|}
\hline \multirow[t]{2}{*}{ Adverse events } & \multicolumn{3}{|c|}{ Availability } & \multicolumn{2}{|l|}{ Effect } & \multicolumn{2}{|c|}{ Heterogeneity } \\
\hline & Trials $(N)$ & $\begin{array}{c}\text { Cases } \\
\text { (events/ } \\
\text { total) }\end{array}$ & $\begin{array}{c}\text { Control } \\
\text { (events/ } \\
\text { total) }\end{array}$ & OR (95\% CI) & $P$ value & $\mathbf{I}^{2}$ & $P$ value \\
\hline \multicolumn{8}{|l|}{ Acute adverse events } \\
\hline Anemia & 8 & $38 / 948$ & $14 / 602$ & $1.87(1.04,3.34)$ & 0.036 & 7.8 & 0.370 \\
\hline Dermatitis & 6 & $28 / 986$ & $31 / 646$ & $0.83(0.50,1.38)$ & 0.474 & 44.1 & 0.111 \\
\hline Mucositis & 7 & $163 / 1020$ & $140 / 672$ & $1.02(0.76,1.37)$ & 0.914 & 69.1 & 0.004 \\
\hline Nausea/vomiting & 8 & $52 / 1034$ & $57 / 688$ & $0.71(0.36,1.41)$ & 0.325 & 53.6 & 0.035 \\
\hline Thrombocytopenia & 7 & $39 / 1020$ & $7 / 672$ & $3.67(1.85,7.23)$ & 0.000 & 14.6 & 0.319 \\
\hline Leukopenia & 6 & $127 / 462$ & $68 / 398$ & $2.13(1.00,4.57)$ & 0.050 & 70.8 & 0.004 \\
\hline Nephrotoxicity & 6 & $11 / 806$ & $10 / 531$ & $0.81(0.35,1.86)$ & 0.613 & 0.0 & 0.936 \\
\hline Fatigue & 3 & $17 / 183$ & $6 / 182$ & $2.47(1.03,5.94)$ & 0.043 & 49.4 & 0.139 \\
\hline Hepatotoxicity & 3 & $5 / 243$ & $3 / 241$ & $1.44(0.43,4.89)$ & 0.581 & 0.0 & 0.876 \\
\hline Xerostomia & 4 & $11 / 305$ & $13 / 243$ & $0.87(0.39,1.92)$ & 0.730 & 29.7 & 0.234 \\
\hline Esophagitis & 2 & $19 / 149$ & $28 / 156$ & $0.69(0.36,1.30)$ & 0.251 & 0.0 & 0.698 \\
\hline Hoarseness & 2 & $6 / 149$ & $6 / 156$ & $1.05(0.36,3.05)$ & 0.934 & 0.0 & 0.535 \\
\hline Neurotoxicity & 3 & $0 / 163$ & $1 / 172$ & $0.65(0.08,4.99)$ & 0.676 & 0.0 & 0.884 \\
\hline Anorexia & 2 & $4 / 77$ & $4 / 86$ & $1.08(0.28,4.20)$ & 0.911 & 0.0 & 0.450 \\
\hline Electrolyte disturbance & 2 & $12 / 572$ & $12 / 290$ & $0.67(0.28,1.57)$ & 0.353 & 0.0 & 0.367 \\
\hline Diarrhea & 2 & $2 / 77$ & $0 / 86$ & $3.37(0.34,33.27)$ & 0.298 & 0.0 & 0.533 \\
\hline Dysphagia & 3 & $21 / 615$ & $17 / 350$ & $1.00(0.52,1.92)$ & 0.991 & 9.8 & 0.330 \\
\hline Infection & 2 & $3 / 601$ & $5 / 334$ & $0.43(0.12,1.52)$ & 0.190 & 0.0 & 0.320 \\
\hline Weigh loss & 3 & $21 / 625$ & $23 / 350$ & $0.92(0.48,1.77)$ & 0.806 & 0.0 & 0.744 \\
\hline Otitis & 2 & $3 / 152$ & $2 / 159$ & $1.36(0.30,6.20)$ & 0.695 & 0.0 & 0.920 \\
\hline \multicolumn{8}{|l|}{ Late adverse events } \\
\hline Xerostomia & 2 & $15 / 120$ & $17 / 112$ & $0.70(0.33,1.52)$ & 0.373 & 14.3 & 0.280 \\
\hline Mucositis & 2 & $3 / 123$ & $2 / 114$ & $1.08(0.04,30.56)$ & 0.962 & 58.8 & 0.119 \\
\hline Otitis & 2 & $10 / 572$ & $9 / 290$ & $0.60(0.25,1.42)$ & 0.248 & 37.0 & 0.208 \\
\hline Subcutaneous fibrosis & 2 & $9 / 120$ & $3 / 112$ & $2.28(0.66,7.82)$ & 0.194 & 0.0 & 0.545 \\
\hline Dermatitis & 2 & $4 / 120$ & $5 / 112$ & $0.66(0.19,2.35)$ & 0.522 & 0.0 & 0.827 \\
\hline Esophagitis & 2 & $1 / 121$ & $0 / 112$ & $1.68(0.15,19.36)$ & 0.676 & 0.0 & 0.748 \\
\hline
\end{tabular}

AC, adjuvant chemotherapy; CCRT, concurrent chemoradiotherapy; CI, confidence interval; NACT, neoadjuvant chemotherapy; OR, odds ratio.

AC might be real in locoregionally advanced nasopharyngeal carcinoma. We confirmed an improvement in PFS and OS by the addition of NACT before CCRT. We also showed benefits in DFFS favoring the addition of NACT, whereas no benefit in locoregional control was shown.
Consistent with previous studies [5-6], this metaanalysis showed that the addition of NACT before CCRT significantly improved PFS. The improvement in PFS was consistent across all trials [9, 13-20], despite a marked variability in terms of NACT regimen, duration 
Table 5: Pair-wise comparisons of neoadjuvant chemotherapy followed by concurrent chemoradiotherapy versus concurrent chemoradiotherapy with or without adjuvant chemotherapy for locoregionally advanced nasopharyngeal carcinoma in the previous and current meta-analyses

\begin{tabular}{|c|c|c|c|c|c|c|c|c|c|c|c|c|}
\hline \multirow{2}{*}{$\begin{array}{l}\text { Meta- } \\
\text { analysis }\end{array}$} & \multicolumn{3}{|c|}{ Overall survival } & \multicolumn{3}{|c|}{ Progression-free survival } & \multicolumn{3}{|c|}{ Localregional failure-free survival } & \multicolumn{3}{|c|}{ Distant failure-free survival } \\
\hline & $\begin{array}{c}\text { Trials } \\
(N ; \text { size })\end{array}$ & $\begin{array}{c}\text { HR } \\
(95 \% \mathrm{CI})\end{array}$ & $\begin{array}{l}\text { Heterogeneity } \\
\left(\mathbf{I}^{2}, \%\right)\end{array}$ & $\begin{array}{c}\text { Trials } \\
(N, \text { size })\end{array}$ & $\begin{array}{c}\text { HR } \\
(95 \% \mathrm{CI})\end{array}$ & $\begin{array}{l}\text { Heterogeneity } \\
\left(\mathbf{I}^{2}, \%\right)\end{array}$ & $\begin{array}{c}\text { Trials } \\
(N, \text { size })\end{array}$ & $\begin{array}{c}\text { HR } \\
(95 \% \mathrm{CI})\end{array}$ & $\begin{array}{l}\text { Heterogeneity } \\
\left(\mathbf{I}^{2}, \%\right)\end{array}$ & $\begin{array}{c}\text { Trials } \\
(N, \text { size })\end{array}$ & $\begin{array}{c}\text { HR } \\
(95 \% \mathrm{CI})\end{array}$ & $\begin{array}{c}\text { Heterogeneity } \\
\left(\mathbf{I}^{2}, \%\right)\end{array}$ \\
\hline $\begin{array}{l}\text { Yan et al., } \\
2015,[8]^{\text {a }}\end{array}$ & $3 ; 378$ & $\begin{array}{c}0.88 \\
(0.57-1.36)\end{array}$ & NI & NI & NI & NI & NI & NI & NI & NI & NI & NI \\
\hline $\begin{array}{l}\text { Song et al., } \\
2015 \text {, [6] }\end{array}$ & $3 ; 318$ & $\begin{array}{c}0.52 \\
(0.21-1.29)\end{array}$ & 61.9 & $4 ; 798$ & $\begin{array}{c}0.66 \\
(0.49-0.90)\end{array}$ & 0.0 & NI & NI & NI & $2 ; 592$ & $\begin{array}{c}0.60 \\
(0.39-0.98)\end{array}$ & 0.0 \\
\hline $\begin{array}{l}\text { Liang et al., } \\
2013,[5]^{\mathrm{b}}\end{array}$ & $3 ; 371$ & $\begin{array}{c}0.99 \\
(0.72-1.36)\end{array}$ & 0.0 & $2 ; 95$ & $\begin{array}{c}0.37 \\
(0.20-0.69)\end{array}$ & 0.0 & $3 ; 347$ & $\begin{array}{c}1.08 \\
(0.84-1.38)\end{array}$ & 34.0 & $2 ; 287$ & $\begin{array}{c}0.98 \\
(0.75-1.27)\end{array}$ & 23.0 \\
\hline $\begin{array}{l}\text { Chen et al., } \\
\text { 2015,[7] }{ }^{a}\end{array}$ & $2 ; 206$ & $\begin{array}{c}0.70 \\
(0.39-1.26)\end{array}$ & 79.0 & NI & NI & NI & $2 ; 206$ & $\begin{array}{c}1.65 \\
(0.95-2.86)\end{array}$ & 0.0 & $2 ; 206$ & $\begin{array}{c}0.51 \\
(0.28-0.95)\end{array}$ & 0.0 \\
\hline $\begin{array}{l}\text { The present } \\
\text { study }{ }^{c}\end{array}$ & $7 ; 1705$ & $\begin{array}{c}0.64 \\
(0.49-0.84)\end{array}$ & 0.0 & $9 ; 2215$ & $\begin{array}{c}0.68 \\
(0.56-0.81)\end{array}$ & 0.0 & $4 ; 591$ & $\begin{array}{c}1.31 \\
(0.83-2.07)\end{array}$ & 0.0 & $5 ; 1177$ & $\begin{array}{c}0.72 \\
(0.53-0.97)\end{array}$ & 0.0 \\
\hline
\end{tabular}

a Shown was the pair-wise comparison included in the network meta-analysis.

${ }^{\mathrm{b}}$ The selected effect size for overall survival, progression-free survival, locoregional failure-free survival, and distant failure-free survival was relative risk with $95 \%$ CIs.

c The selected effect size for locoregional failure-free survival was odds ratio with $95 \%$ CIs.

CI, confidence interval; HR, hazard ratio; NI, not investigated.

of follow-up, radiotherapy technique, method of data extraction, and staging system for nasopharyngeal carcinoma in the included trials. The variability was equivalent to adding "noise" to the analysis. It was likely that an association might be weakened or masked by noise [28]. In spite of the variability, the association between a significant improvement in PFS and NACT was also seen consistently across different patient subgroups, and there was no evidence of heterogeneity in all the meta-analyses. The extensive consistency provided optimal evidence of the credibility of an association [29] between NACT and an improved PFS. In addition, the credibility could be further strengthened by the clinical variability. Therefore, our meta-analysis provided strong evidence that NACT followed by CCRT improved PFS when compared to CCRT with or without $\mathrm{AC}$ in patients with locoregionally advanced nasopharyngeal carcinoma.

The results of this meta-analysis of OS were related with two important clinical issues. First, NACT followed by CCRT had shown a marked improvement in OS compared versus CCRT with or without AC. The present meta-analysis had the largest sample size up-to-date and the sufficient statistical power to detect the treatment effect, whereas previous metaanalyses failed to do so [5-8]. This was in line with the results of our subgroup analyses: no significant benefit in OS in all small patient subgroups ( $\mathrm{r}<$ 800 ), but a consistently significant benefit in OS in all large patient subgroups $(\mathrm{n}>800)$. We identified potential evidence of heterogeneity when trials were stratified based on NACT regimens (two-drug NACT or three-drug NACT). Two-drug NACT regimen was shown to be better in OS compared with three-drug NACT. Although the difference was not statistically significant, the power of this test was low [30], and a genuine difference could not be ruled out. Because this result was opposite to that found for head and neck cancer [31-33], but was in agreement with that found for lung cancer [34-35]. This proposed the second important clinical issue. This issue might be solved by two ongoing randomized, controlled Phase III trials (NCT01536223: docetaxel plus cisplatin and 5-fluorouracil versus cisplatin and 5-fluorouracil; NCT02016417: docetaxel plus cisplatin and 5-Fluorouracil versus gemcitabine and cisplatin).

Our meta-analysis showed an improvement in DFFS, but not in the incidence of locoregional failure, with the incorporation of NACT. These results were in line with previous reports $[6,36]$. Regarding DFFS, the leave-1-out sensitivity analysis showed that the positive finding relied heavily on the trial conducted by $\mathrm{Ma}$ et al. [14]. The meta-analysis of the remaining trials was unable to show a significantly improved DFFS favoring NACT, but each individual trial consistently showed such a trend. These could be an indication of a small but a real benefit in DFFS. Furthermore, Ma et al.'s trial was a large and well-designed multicenter randomized controlled study (ClinicalTrials.gov, NCT01245959) [14], the inclusion of this trial was necessary. Taken together, these suggested a genuine benefit in DFFS favoring NACT for locoregionally advanced nasopharyngeal carcinoma.

A concern for the addition of NACT is an increase of severe acute adverse events, which in turn compromises the delivery of subsequent CCRT. The present meta-analysis showed that the addition of NACT was mainly associated with an increased risk of severe hematologic adverse events, including leukopenia and thrombocytopenia. These acute adverse events were uncomplicated and manageable with growth factor support. 
This meta-analysis had several limitations: (1) Due to the lack of individual patient data, we were not able to check each trial to apply consistent conditions for inclusion and to standardize analysis techniques in this meta-analysis. However, literature based meta-anlayses are often consistent with those based on individual patient data [37], and should not be viewed as "inferior." [38]. (2) Although the tests for publication bias did not identify major publication bias, these might not have enough power unless the number of included studies was more than 10 [39]. Our subgroup analyses indicated that small studies did not show significantly larger effects than large studies. In addition, according to the Venice criteria that were developed to assess cumulative evidence of genetic associations, a small effect size (such as, OR $<1.15$ or $>0.87$ ) might be vulnerable to biases [29, 40-41]. Our meta-analyses showed much larger effect sizes (a HR of 0.68 for PFS and a HR of 0.64 for OS). These results suggested that publication bias might not be a significant threat to our meta-analyses. (3) The duration of followup was short in most trials included in this meta-analysis. Although there is evidence that PFS is predictive of OS for a number of cancer types (nasopharyngeal carcinoma [11] and bladder cancer [42]), a re-analysis of the data after longer follow-up will enable us to better assess the treatment effect on OS. (4) Due to the incomplete data inclusion, the results of the meta-analyses on DFFS and locoregional failure should be viewed as exploratory only. The use of locoregional failure rate to compute OR instead of the use of HR might result in bias [43]. (5) Regarding most adverse events, especially late adverse events, the large amount of missing data did not allow for any meaningful analysis.

In conclusion, this meta-analysis confirmed that NACT followed by CCRT provided a significantly improved PFS compared versus CCRT with or without AC in patients with locoregionally advanced nasopharyngeal carcinoma. We also showed that the addition of NACT was associated with a significant benefit in OS. Although longer follow-up is needed for a better assessment of $\mathrm{OS}$, it is reasonable to recommend the addition of NACT to CCRT for patients with locoregionally advanced nasopharyngeal carcinoma.

\section{MATERIALS AND METHODS}

\section{Selection criteria}

This meta-analysis was done based on a prespecified protocol. To be eligible, trials needed to compare NACT plus CCRT versus CCRT with or without AC in previously untreated patients with histologically proven nasopharyngeal carcinoma without distant metastases. NACT, CCRT, and AC were defined as chemotherapy administered before, during, and after radiotherapy, respectively. Only randomized controlled trials were eligible for inclusion. Trials needed to provide data on PFS. Trials without sufficient data for quantitative estimates were listed in the summary overview but were not subjected to a meta-analysis. According to the Cochrane Handbook for Systematic Reviews of Interventions, including trials at high risk of bias may lower the quality of evidence in a metaanalysis [27]. Therefore, risk of bias for each identified trial was assessed, and trials at high risk of bias were excluded. Because patients with locoregionally advanced nasopharyngeal carcinoma who received CCRT had a twoyear survival rate of more than $90 \%[22,44]$, a minimum of 2 years follow-up was required for the meta-analysis of OS. Published and unpublished trials were eligible. There were no language restrictions. Case reports, editorials, metaanalyses, and review articles were excluded.

\section{Literature search strategy}

Following the Cochrane Handbook for Systematic Reviews of Interventions [27] and the Preferred Reporting Items for Systematic Reviews and Meta-Analyses guidelines [45], we conducted a comprehensive search of the literature before November 13, 2015. The following databases were used: PubMed, Web of Science, ClinicalTrials.gov, EMBASE, and Chinese National Knowledge Infrastructure databases. A search of the Proceedings of the Annual Meetings of the American Society of Clinical Oncology, American Association for Cancer Research, American Society for Radiation Oncology, European Society for Medical Oncology/European Cancer Organisation, European Society for Radiotherapy \& Oncology, Chinese Society of Clinical Oncology, and Chinese Society of Radiation Oncology was conducted to identify relevant studies published in abstract form. In addition, we manually screened citation lists of the retrieved articles to ensure a wider search. The following search terms were used: (chemotherapy) AND (nasopharyngeal OR nasopharynx) AND (cancer OR carcinoma OR neoplasm OR tumor OR malignancy OR malignant) AND radiotherapy.

\section{Data extraction}

Two investigators extracted the following data independently from each individual trial: first author, publication year, region where research was conducted, ethnicity, number of patients, histologic type (WHO criteria), TNM stage, follow-up duration, treatment protocol, compliance with treatment, response to treatment, exclusion (yes/no) from trial analysis and reason for exclusion, and failure pattern.

$\mathrm{HR}$ and its $95 \% \mathrm{CI}$ were directly used if these values were reported in a trial. Otherwise, two investigators calculated the data independently according to the method by Parmar et al. [46] Whenever there were missing data, study authors were contacted via e-mail. Disagreement was resolved by discussion between authors. 


\section{Risk of bias assessments}

Risk of bias was independently assessed by two authors, based on the guidelines outlined in the Cochrane Handbook for Systematic Reviews of Interventions Version 5.1.0 [27]. Disagreements were resolved by consensus with a third author. Briefly, each trial was assessed for the following domains: random sequence generation, allocation concealment, blinding of participants and personnel, blinding of outcome assessment, incomplete outcome data, selective reporting, and other sources of bias. Each domain was defined as having a low, high, or unclear risk for bias. It was impossible to blind study participants and personnel to whether or not NACT had been undertaken, and how this influenced the outcome of the clinical trial was not known. Therefore, the domain for all trials was categorized as unclear risk of bias. A trial was considered to have a low risk of bias if all criteria were "low", an unclear risk of bias if any criteria were "unclear", and a high risk of bias if any criteria were "high".

\section{Subgroup analysis and sensitivity analysis}

If there was more than 6 trials included in a metaanalysis, subgroup analyses were conducted. The subgroup analysis was aimed at exploring whether the treatment effect of NACT was stable or dependent on features of the included trials. For this purpose, we predefined the following subgroups based on: duration of follow-up, radiotherapy technique (conventional radiotherapy versus 3D-CRT or IMRT), NACT regimen (two drugs versus three drugs; taxol-included and non-taxol-included), CCRT timing (q1wk versus q3wk), sample size, method of data extraction (directly reported versus indirectly extracted), source of data (published versus unpublished), and $\mathrm{AC}$ (with versus without).

For sensitivity analysis, we excluded 1 trial at a time and analyzed the remaining trials to explore whether the results were influenced by a particular trial.

\section{Outcomes}

The main study end point was PFS. Secondary end points were OS, DFFS, the incidence of locoregional failure, and the incidence of treatment-related adverse events. All time-to-event variables were calculated from date of randomization. PFS was defined as time to date of progression (locoregional failure or distant failure) or death (whichever occurred first). OS was defined as time to date of death from any cause. DFFS was defined as time to date of distant failure. If a distant failure and a locoregional failure occurred in a patient at the same time, the patient was considered as having an event for a distant failure only.

\section{Statistical analysis}

An intent-to-treat analysis was applied for this metaanalysis [47]. HRs with 95\% CIs were used to express results regarding PFS, OS, and DFFS. HRs were calculated using a fixed-effect model. A HR less than 1 suggested an improved survival for NACT plus CCRT treatment compared versus CCRT with or without AC treatment. For locoregional failure and treatment-related adverse events, most reports provided only the absolute number of events, and there was no information available to calculate the HR. Therefore, odds ratios (ORs) was used as the summary statistic for the comparison between groups. The heterogeneity between trials was investigated by using the Cochrane $\mathrm{Q}$ test and the $\mathrm{I}^{2}$ statistic. A random-effects model (DerSimonian and Laird method [48]) was used in case of obvious heterogeneity (the $P$ value of the Cochrane $\mathrm{Q}$ test was $<0.10$ or the $\mathrm{I}^{2}$ value was $>50 \%$ ); otherwise, a fixed-effects model (Mantel and Haenszel [49]) was applied. The test of interaction proposed by Altman et al. [30] was used to compare differences in treatment effect across subgroups. Publication bias was evaluated by visualizing the symmetry of the funnel plot and by Begg's rank correlation test and Egger's linear regression test. [50-51] We used Stata software (StataCorp), version 12. Statistical significance was defined as a $P$ value of $<0.05$ (two-sided).

\section{ACKNOWLEDGMENTS}

The authors thank Dr. Anne W.M. Lee and his group, Clinical Oncology Center, The University of Hong Kong-Shenzhen Hospital, Shenzhen,Guangdong Province, China, for their kindness in providing us with the necessary data for this meta-analysis. We thank Dr Hua He (Cancer Center, the First Hospital of Jilin University, Changchun, Jilin, China) for her statistical assistance.

\section{CONFLICTS OF INTEREST}

There is no conflict of interest.

\section{GRANT SUPPORT}

This work was supported by Natural Science Foundation of Science and Technology Agency of Jilin Province (Bethune special foundation), China (Grant No. 20160101098JC to HFJ).

\section{REFERENCES}

1. National Comprehensive Cancer Network. Practice guidelines in oncology - version V.1.2015 (Head and Neck Cancers)updated 2015; http://www.ncen.org/professionals/ physician_gls/pdf/head-and-neck.pdf (6 September 2015, date last accessed)

2. Chen YP, Wang ZX, Chen L, Liu X, Tang LL, Mao YP, Li WF, Lin AH, Sun Y and Ma J. A Bayesian network metaanalysis comparing concurrent chemoradiotherapy followed 
by adjuvant chemotherapy, concurrent chemoradiotherapy alone and radiotherapy alone in patients with locoregionally advanced nasopharyngeal carcinoma. Ann Oncol. 2015; 26:205-211.

3. Blanchard P, Lee A, Marguet S, Leclercq J, Ng WT, Ma J, Chan AT, Huang PY, Benhamou E, Zhu G, Chua DT, Chen Y, Mai HQ, Kwong DL, Cheah SL, Moon J, et al. Chemotherapy and radiotherapy in nasopharyngeal carcinoma: an update of the MAC-NPC meta-analysis. Lancet Oncol. 2015; 16:645-655.

4. OuYang PY, Xie C, Mao YP, Zhang Y, Liang XX, Su Z, Liu Q and Xie FY. Significant efficacies of neoadjuvant and adjuvant chemotherapy for nasopharyngeal carcinoma by meta-analysis of published literature-based randomized, controlled trials. Ann Oncol. 2013; 24:2136-2146.

5. Liang ZG, Zhu XD, Tan AH, Jiang YM, Qu S, Su F and $\mathrm{Xu}$ GZ. Induction chemotherapy followed by concurrent chemoradiotherapy versus concurrent chemoradiotherapy with or without adjuvant chemotherapy for locoregionally advanced nasopharyngeal carcinoma: meta-analysis of 1,096 patients from 11 randomized controlled trials. Asian Pac J Cancer Prev. 2013; 14:515-521.

6. Song Y, Wang W, Tao G and Zhou X. Survival benefit of induction chemotherapy in treatment for locally advanced nasopharyngeal carcinoma--A time-to-event meta-analysis. Oral Oncol. 2015; 51:764-769.

7. Chen YP, Guo R, Liu N, Liu X, Mao YP, Tang LL, Zhou GQ, Lin AH, Sun Y and Ma J. Efficacy of the Additional Neoadjuvant Chemotherapy to Concurrent Chemoradiotherapy for Patients with Locoregionally Advanced Nasopharyngeal Carcinoma: a Bayesian Network Meta-analysis of Randomized Controlled Trials. J Cancer. 2015; 6:883-892.

8. Yan M, Kumachev A, Siu LL and Chan KK. Chemoradiotherapy regimens for locoregionally advanced nasopharyngeal carcinoma: A Bayesian network metaanalysis. Eur J Cancer. 2015; 51:1570-1579.

9. Fountzilas G, Ciuleanu E, Bobos M, Kalogera-Fountzila A, Eleftheraki AG, Karayannopoulou G, Zaramboukas T, Nikolaou A, Markou K, Resiga L, Dionysopoulos D, Samantas E, Athanassiou H, Misailidou D, Skarlos D and Ciuleanu T. Induction chemotherapy followed by concomitant radiotherapy and weekly cisplatin versus the same concomitant chemoradiotherapy in patients with nasopharyngeal carcinoma: a randomized phase II study conducted by the Hellenic Cooperative Oncology Group (HeCOG) with biomarker evaluation. Ann Oncol. 2012; 23:427-435.

10. Chan AT, Leung SF, Ngan RK, Teo PM, Lau WH, Kwan WH, Hui EP, Yiu HY, Yeo W, Cheung FY, Yu KH, Chiu KW, Chan DT, Mok TS, Yau S, Yuen KT, et al. Overall survival after concurrent cisplatin-radiotherapy compared with radiotherapy alone in locoregionally advanced nasopharyngeal carcinoma. J Natl Cancer Inst. 2005; 97:536-539.
11. Chen YP, Sun Y, Chen L, Mao YP, Tang LL, Li WF, Liu X, Zhang WN, Zhou GQ, Guo R, Lin AH and Ma J. Surrogate endpoints for overall survival in combined chemotherapy and radiotherapy trials in nasopharyngeal carcinoma: Metaanalysis of randomised controlled trials. Radiother Oncol. 2015; 116:157-166.

12. Adelstein DJ. Clinical trial design in head and neck cancer: what has the oncologist learned? Lancet Oncol. 2012; 13:e318-323.

13. Lee AW, Ngan RK, Tung SY, Cheng A, Kwong DL, Lu TX, Chan AT, Chan LL, Yiu H, Ng WT, Wong F, Yuen KT, Yau S, Cheung FY, Chan OS, Choi H, et al. Preliminary results of trial NPC-0501 evaluating the therapeutic gain by changing from concurrent-adjuvant to inductionconcurrent chemoradiotherapy, changing from fluorouracil to capecitabine, and changing from conventional to accelerated radiotherapy fractionation in patients with locoregionally advanced nasopharyngeal carcinoma. Cancer. 2015; 121:1328-1338.

14. Ma J, Chen N, Zhang N, Hu G, Sun Y, Chen X, Li J, Zhu $\mathrm{X}$, Hui C, Xu X, Sun Y and Xie F. Induction chemotherapy plus concurrent chemoradiotherapy versus concurrent chemoradiotherapy alone in patients with locoregionally advanced nasopharyngeal carcinoma:preliminary results of a phase 3 multicentre randomised controlled trial. Eur J Cancer. 2014; 50:e3-e4.

15. Gao J, Gao T, Dong Z, Dong G, Li G, Wu H and Fan X. A prospective and randomized study of induction chemotherapy combined with concurrent chemoradiotherapy in the treatment for nasopharyngeal carcinoma stage T3-4N2-3M0. Chin J Clin Oncol. 2013; 19:161-165.

16. Sun R. A phase II study of induction chemotherapy plus concurrent chemoradiotherapy compared with concurrent chemoradiotherapy alone for patients with localregionally advanced nasopharyngeal carcinoma. MS thesis, Oncology Department, Sun Yat-sen University Cancer Centre, Guangzhou, China, 2009.

17. Huang S, Deng G, Huang G, Li Y, Meng Y and Chen J. Efficacy of induction chemotherapy combined with concurrent chemoradiotherapy for advanced nasopharyngeal carcinoma. Chin J Clin Oncol. 2012; 39:788-791.

18. Tan T, Lim WT, Fong KW, Cheah SL, Soong YL, Ang MK, Ng QS, Tan D, Ong WS, Tan SH, Yip C, Quah D, Soo KC and Wee J. Concurrent chemo-radiation with or without induction gemcitabine, Carboplatin, and Paclitaxel: a randomized, phase $2 / 3$ trial in locally advanced nasopharyngeal carcinoma. Int J Radiat Oncol Biol Phys. 2015; 91:952-960.

19. Hui EP, Ma BB, Leung SF, King AD, Mo F, Kam MK, Yu BK, Chiu SK, Kwan WH, Ho R, Chan I, Ahuja AT, Zee $\mathrm{BC}$ and Chan AT. Randomized phase II trial of concurrent cisplatin-radiotherapy with or without neoadjuvant docetaxel and cisplatin in advanced nasopharyngeal carcinoma. J Clin Oncol. 2009; 27:242-249.

20. Ruste SA, Dizon DS, Ramirez F, Sagpao C, Tiangco B and Fragante E. Induction chemotherapy followed by concurrent 
chemoradiotherapy vs concurrent chemoradiotherapy followed by chemotherapy in the treatment of patients with advanced nasopharyngeal carcinoma. Phillippine Journal of Internal Medicine. 2011; 49:1-7.

21. Xu MJ, Ye JM, Wu LQ and Wang XC. Neoadjuvant chemotherapy using DPF followed by radiotherapy concurrent chemoradiotherapy with cisplatin in the Treatment for locally advanced nasopharyngeal carcinoma. Journal of Practical Oncology. 2011; 26:291-294.

22. Guo LB and Song WS. Neoadjuvant chemotherapy using TP followed by concurrent chemoradiotherapy for locally advanced nasopharyngeal carcinoma. Guangzhou Medical Journal. 2010; 41:9-10.

23. Chen XJ, Qiu RL, Yang CL and L YQ. Study of TPF neoadjuvant chemotherapy followed by concurrent intensity modulated radiochemotherapy in the treatment for 30 cases with locally advanced nasopharyngeal carcinoma. Chin J Clin Oncol Rehabil. 2012; 19:201-204.

24. He AJ. Neoadjuvant chemotherapy followed by concurrent chemoradiotherapy in the treatment of locally advanced nasopharyngeal carcinoma. Hebei Med J. 2011; Vol 33 , No. 14:2115-2116.

25. He QD, Yang L, Nie YH and Kang HD. A phaseII study of neoadjuvant chemotherapy followed by concurrent chemoradiotherapy in locally advanced nasopharyngeal carcinoma. Chinese Clinical Oncology. 2009; 14:1011-1014.

26. Zheng QP, Shi CL, Zhou YY, Li ZH, Zhang SX and Meng YZ. Comparative analysis of induction chemotherapy plus concurrent chemoradiotherapy versus concurrent chemoradiotherapy alone for locally advanced nasopharyngeal carcinoma. J Shanxi Med Univ. 2013; 44:824-826.

27. Higgins J GS, eds. . Cochrane handbook for systematic reviews of interventions version 5.1.0, updated March, 2011. Oxford, UK: The Cochrane Collaboration, 2011. http://handbook.cochrane.org (accessed June 8, 2015).

28. De Laurentiis M, Arpino G, Massarelli E, Ruggiero A, Carlomagno C, Ciardiello F, Tortora G, D’Agostino D, Caputo F, Cancello G, Montagna E, Malorni L, Zinno L, Lauria R, Bianco AR and De Placido S. A meta-analysis on the interaction between HER-2 expression and response to endocrine treatment in advanced breast cancer. Clin Cancer Res. 2005; 11:4741-4748.

29. Ioannidis JP, Boffetta P, Little J, O'Brien TR, Uitterlinden AG, Vineis P, Balding DJ, Chokkalingam A, Dolan SM, Flanders WD, Higgins JP, McCarthy MI, McDermott DH, Page GP, Rebbeck TR, Seminara $\mathrm{D}$, et al. Assessment of cumulative evidence on genetic associations: interim guidelines. Int J Epidemiol. 2008; 37:120-132.

30. Altman DG and Bland JM. Interaction revisited: the difference between two estimates. BMJ. 2003; 326:219.
31. Lorch JH, Goloubeva O, Haddad RI, Cullen K, Sarlis N, Tishler R, Tan M, Fasciano J, Sammartino DE and Posner MR. Induction chemotherapy with cisplatin and fluorouracil alone or in combination with docetaxel in locally advanced squamous-cell cancer of the head and neck: long-term results of the TAX 324 randomised phase 3 trial. Lancet Oncol. 2011; 12:153-159.

32. Pointreau Y, Garaud P, Chapet S, Sire C, Tuchais C, Tortochaux J, Faivre S, Guerrif S, Alfonsi M and Calais G. Randomized trial of induction chemotherapy with cisplatin and 5-fluorouracil with or without docetaxel for larynx preservation. J Natl Cancer Inst. 2009; 101:498-506.

33. Blanchard P, Bourhis J, Lacas B, Posner MR, Vermorken JB, Hernandez JJ, Bourredjem A, Calais G, Paccagnella A, Hitt R and Pignon JP. Taxane-cisplatin-fluorouracil as induction chemotherapy in locally advanced head and neck cancers: an individual patient data meta-analysis of the meta-analysis of chemotherapy in head and neck cancer group. J Clin Oncol. 2013; 31:2854-2860.

34. Pirker R. Two- versus three-drug combinations in the chemotherapy of advanced non-small-cell lung cancer. Lung Cancer. 2002; 38:S53-55.

35. National Comprehensive Cancer Network Practice guidelines in oncology - version V12015 (Non-Small Cell Lung Cancer)updated 2015; http://wwwnccnorg/ professionals/physician_gls/pdf/nsclpdf (6 September 2015, date last accessed).

36. Sun X, Zeng L, Chen C, Huang Y, Han F, Xiao W, Liu $\mathrm{S}$ and $\mathrm{Lu} \mathrm{T}$. Comparing treatment outcomes of different chemotherapy sequences during intensity modulated radiotherapy for advanced $\mathrm{N}$-stage nasopharyngeal carcinoma patients. Radiat Oncol. 2013; 8:265.

37. Steinberg KK, Smith SJ, Stroup DF, Olkin I, Lee NC, Williamson GD and Thacker SB. Comparison of effect estimates from a meta-analysis of summary data from published studies and from a meta-analysis using individual patient data for ovarian cancer studies. Am J Epidemiol. 1997; 145:917-925.

38. Huncharek M and Kupelnick B. In regards to Baujat et al. Chemotherapy in locally advanced nasopharyngeal carcinoma: An individual patient data meta-analysis of eight randomized trials and 1753 patients (Int J Radiat Oncol Biol Phys 2006;64:47-56). Int J Radiat Oncol Biol Phys. 2006; 65:958; author reply 958-959.

39. Macaskill P, Walter SD and Irwig L. A comparison of methods to detect publication bias in meta-analysis. Stat Med. 2001; 20:641-654.

40. Vineis P, Manuguerra M, Kavvoura FK, Guarrera S, Allione A, Rosa F, Di Gregorio A, Polidoro S, Saletta F, Ioannidis JP and Matullo G. A field synopsis on low-penetrance variants in DNA repair genes and cancer susceptibility. J Natl Cancer Inst. 2009; 101:24-36.

41. Chatzinasiou F, Lill CM, Kypreou K, Stefanaki I, Nicolaou V, Spyrou G, Evangelou E, Roehr JT, Kodela E, Katsambas A, Tsao H, Ioannidis JP, Bertram L and Stratigos AJ. 
Comprehensive field synopsis and systematic meta-analyses of genetic association studies in cutaneous melanoma. $\mathrm{J}$ Natl Cancer Inst. 2011; 103:1227-1235.

42. Winquist E, Kirchner TS, Segal R, Chin J and Lukka H. Neoadjuvant chemotherapy for transitional cell carcinoma of the bladder: a systematic review and meta-analysis. J Urol. 2004; 171:561-569.

43. Michiels S, Piedbois P, Burdett S, Syz N, Stewart L and Pignon JP. Meta-analysis when only the median survival times are known: a comparison with individual patient data results. Int J Technol Assess Health Care. 2005; 21:119-125.

44. Kong L, Hu C, Niu X, Zhang Y, Guo Y, Tham IW and Lu JJ. Neoadjuvant chemotherapy followed by concurrent chemoradiation for locoregionally advanced nasopharyngeal carcinoma: interim results from 2 prospective phase 2 clinical trials. Cancer. 2013; 119:4111-4118.

45. Liberati A, Altman DG, Tetzlaff J, Mulrow C, Gotzsche PC, Ioannidis JP, Clarke M, Devereaux PJ, Kleijnen J and Moher D. The PRISMA statement for reporting systematic reviews and meta-analyses of studies that evaluate health care interventions: explanation and elaboration. PLoS Med. 2009; 6:e1000100.
46. Parmar MK, Torri V and Stewart L. Extracting summary statistics to perform meta-analyses of the published literature for survival endpoints. Stat Med. 1998; 17:2815-2834.

47. Baujat B, Audry H, Bourhis J, Chan AT, Onat H, Chua DT, Kwong DL, Al-Sarraf M, Chi KH, Hareyama M, Leung SF, Thephamongkhol K and Pignon JP. Chemotherapy in locally advanced nasopharyngeal carcinoma: an individual patient data meta-analysis of eight randomized trials and 1753 patients. Int J Radiat Oncol Biol Phys. 2006; 64:47-56.

48. DerSimonian R and Laird N. Meta-analysis in clinical trials. Control Clin Trials. 1986; 7:177-188.

49. Mantel $\mathrm{N}$ and Haenszel W. Statistical aspects of the analysis of data from retrospective studies of disease. J Natl Cancer Inst. 1959; 22:719-748.

50. Begg CB and Mazumdar M. Operating characteristics of a rank correlation test for publication bias. Biometrics. 1994; 50:1088-1101.

51. Egger M, Davey Smith G, Schneider M and Minder C. Bias in meta-analysis detected by a simple, graphical test. BMJ. 1997; 315:629-634. 